\title{
CARACTERÍSTICAS PSICOMOTORAS E SENSORIAIS DE CRIANÇAS COM TRANSTORNO DO ESPECTRO AUTISTA (TEA) EM ATENDIMENTO TERAPÊUTICO OCUPACIONAL
}

\author{
CARACTERÍSTICAS PSICOMOTORAS Y SENSORIALES DE NIÑOS CON TRASTORNO \\ DEL ESPECTRO AUTISTICO (TEA) EN ATENCIÓN DE TERAPIA OCUPACIONAL \\ PSYCHOMOTOR AND SENSORY CHARACTERISTICS OF CHILDREN WITH AUTISTIC \\ SPECTRUM DISORDER (TEA) IN OCCUPATIONAL THERAPY INTERVENTION
}

\section{Amanda Dourado Souza Akahosi Fernandes'1, Letícia Migliatti Polli², Luciana Bolzan Agnelli Martinez ${ }^{3}$}

\begin{abstract}
RESUMO
Atualmente o autismo tem sido classificado como Transtorno do Espectro Autista (TEA), podendo apresentar como critério diagnóstico os atrasos no desenvolvimento psicomotor e alterações sensoriais, além das características típicas. Objetivo: Identificar as características psicomotoras e sensoriais de crianças com diagnóstico de TEA atendidas no setor de Terapia Ocupacional na Unidade Saúde Escola da Universidade Federal de São Carlos (USE-UFSCar). Método: Trata-se de uma pesquisa exploratória e descritiva, de abordagem quantitativa. Participaram nove crianças de quatro a dez anos de idade com diagnóstico de TEA, e seus responsáveis. Os instrumentos utilizados para coleta de dados foram um Questionário de dados gerais da criança; Perfil sensorial e Bateria Psicomotora. A coleta dos dados ocorreu na própria Unidade,sendo que os dados dos instrumentos padronizados foram analisados a partir das instruções pré-existentes, e os dados do Questionário de forma descritiva. Resultados: Identificou-se que as crianças participantes apresentam déficits psicomotores nos seguintes aspectos: noção do corpo, estruturação espaço-tempo, praxia global e fina. Em relação ao sistema sensorial, as alterações identificadas no presente estudo foram na audição, sistema vestibular e multissensorial, constante procura sensorial, inatenção e na motricidade fina. Conclusão: Aponta-se para a importância de maiores investimentos nesse campo, uma vez que pode favorecer as intervenções voltadas a esse público.
\end{abstract}

\section{PALAVRAS-CHAVE}

Transtorno autístico; criança;transtornos das sensações; limiar sensorial, desenvolvimento infantil.

1 Doutora em Terapia Ocupacional pelo Programa de Pós Graduação em Terapia Ocupacional da Universidade Federal de São Carlos. Professora Assistente A do Departamento de Terapia Ocupacional, Universidade Federal de São Carlos, São Carlos, São Paulo, Brasil. amanda.d.fernandes@hotmail.com Orcid: 0000-0001-8006-8117. Correio Postal: Universidade Federal de São Carlos, Rodovia Washington Luiz, km 235, Cep 13565-905, São Carlos, São Paulo, Brasil. Número de telefone: +55 (16) 3351-8750.

2 Estudante do Curso de Terapia Ocupacional do Departamento de Terapia Ocupacional, Universidade Federal de São Carlos (UFSCar), São Carlos, São Paulo, Brasil. le_mp@hotmail.com Orcid: 0000-0002-9331-5452

3 Doutora em Ciências pelo Programa de Pós Graduação Interunidades em Bioengenharia EESC/FMRP/IQSC - USP. Professora Adjunta do Departamento de Terapia Ocupacional, Universidade Federal de São Carlos, São Carlos, São Paulo, Brasil. to@luagnelli.com.br Orcid: 0000-0003-0873-9806 


\section{RESUMEN}

Actualmente, el autismo se ha clasificado como trastorno del espectro autista (TEA), y puede presentarse como criterio de diagnóstico de retrasos en el desarrollo psicomotor y cambios sensoriales, además de las características típicas. Objetivo: identificar las características psicomotoras y sensoriales de los niños diagnosticados con TEA tratados en el sector de terapia ocupacional en la Unidad de la Escuela de Salud de la Universidad Federal de São Carlos (USE-UFSCar). Método: Esta es una investigación exploratoria y descriptiva, con un enfoque cuantitativo. Participaron nueve niños de cuatro a diez años diagnosticados con tea y sus padres. Los instrumentos utilizados para la recopilación de datos fueron un cuestionario de datos generales del niño; Perfil sensorial y batería psicomotora. La recopilación de datos tuvo lugar en la propia Unidad, y los datos de los instrumentos estandarizados se analizaron utilizando instrucciones preexistentes y los datos del Cuestionario de manera descriptiva. Resultados: Se identificó que los niños participantes tienen déficits psicomotores en los siguientes aspectos: noción del cuerpo, estructuración espacio-tiempo, praxis globaly fina. Con respecto al sistema sensorial, los cambios identificados en el presente estudio fueron en el sistema auditivo, vestibular y multisensorial, búsqueda sensorial constante, falta de atención y en el motricidad fina. Conclusión: señala la importancia de mayores inversiones en este campo, ya que puede favorecer intervenciones dirigidas a esta audiencia.

\section{PALABRAS CLAVE}

Transtorno autístico, niño; trastornos de la sensación; umbral sensorial, desarrollo infantil.

\section{ABSTRACT}

Currently, autism has been classified as Autism Spectrum Disorder (ASD), and may present as a diagnostic criterion delays in psychomotor development and sensory changes, in addition to the typical characteristics. Objective: To identify the psychomotor and sensory profile of children diagnosed with ASD. Method: This is a survey study, exploratory and descriptive, with a qualitative and quantitative approach. Nine children from four to ten years old diagnosed with a diagnosis of ASD participated, linked to the School Health Unit (USE)-UFSCar and their guardians. The instruments used for data collection were a questionnaire of general data of the child; Sensory profile and Psychomotor Battery. Data collection took place at the Unit itself, and data from standardized instruments were analyzed based on pre-existing instructions, and data from the Questionnaire were analyzed descriptively. Results: Children with ASD have psychomotor deficits in the following aspects: body notion, space-time structuring, global and global and fine praxis. Regarding the sensory system, the changes identified were in hearing, vestibular and multisensory system, constant sensory search, inattention and fine motor. Conclusion: It points to the importance of greater investments in this field, since it can favor interventions aimed at this audience.

\section{KEYWORDS}

Autistic disorder, child, sensation disorders, sensory thresholds, child development.

Recibido: $27 / 01 / 2021$

Aceptado: 28/10/2021 


\section{INTRODUÇÃO}

Até o século XIX, os indivíduo sem sofrimento psíquico que se distanciavam do padrão tido como "normalidade", recebiam o diagnóstico de "idiotia"-termo precursor que englobava as psicoses infantis, o retardo mental, a esquizofrenia e o autismo. Tendo em vista esta classificação, não havia particularidades no diagnóstico e consequentemente no tratamento (Brasil, 2013).

Foi somente a partir de1943 que Leo Kanner descreveu e discriminou pela primeira vez uma nova síndrome denominada de autismo infantil, a partir da identificação de características em comum presentes em um grupo de 11 crianças, tais como-inquietação, pobreza de expressões, movimentos estereotipados, rítmicos e repetitivos e déficit na linguagem (Brasil, 2013).

Porém, aponta-se que o autismo teve seu reconhecimento científico oficial décadas mais tarde,sendo que em 1980 passou a ser considerado como "Transtorno Invasivo do Desenvolvimento" (TID) pela Classificação Internacional de Doenças (CID). A partir deste marco, algumas transformações no que tange ao diagnóstico e sua classificação foram ocorrendo de forma que, em 2013, o Manual Diagnóstico e Estatístico de Transtornos Mentais na sua quinta edição (DSM-V), passou a classificar este quadro como Transtorno do Espectro Autista (TEA) (Brasil, 2013).

Quanto as características, a criança tende a apresentar antes dos três anos, dificuldades na interação social, comportamentos repetitivos e estereotipados e interesses restritos, juntamente com atraso na linguagem e compreensão, podendo surgir outras limitações associadas (Klin, 2006; Lima et al, 2017).

Para além das características clássicas, alterações psicomotoras e sensoriais podem se evidenciar como, por exemplo, no tônus muscular, equilíbrio, coordenação motora, esquema corporal e lateralidade, comprometendo o desenvolvimento (Oliveira et al, 2015).

Nesta direção, pela primeira vez, na quinta edição do DSM, as alterações sensoriais são destacadas dentre os critérios de diagnóstico, indicando que 90\% das crianças podem apresentar alterações sensoriais no campo auditivo, vestibular, proprioceptivo, visual, olfativo e oral (Leal et al, 2015; Posar \& Visconti, 2018). De acordo com este manual,são consideradas características sensoriais associadas ao diagnóstico do TEA, respostas intensas a certos sons e texturas, cheirar ou tocar objetos, fascínio por luzes ou objetos com movimento, e ocasionalmente, indiferença à dor e temperatura. Como consequência, as reações destas crianças estão associadas à repostas hipo ou hiperreativas, comumente presente no quadro (American Psychiatric Association [APA], 2013).

Estudostêm sido realizados visando identificar as possíveis alterações psicomotoras e sensoriais de crianças e adolescentes com tea (Gadia et al., 2004; Gomes et al., 2008; Leal et al., 2015; Miccas et al., 2014; Prestes et al., 2009; Okuda, 2010; Andrade, 2012; Oliveira et al., 2015; Sandroni et al., 2015; Soares \& Cavalcante Neto, 2015; Ferreira, 2016; Gusman, 2017).

Quanto às alterações psicomotoras, Prestes et al. (2009) realizaramum estudo com uma criança de seis anos diagnosticada com TEA, com o objetivo de investigar o seu desenvolvimento motor. Foram realizadostestes pré e pós intervenção por meio da Escala de Desenvolvimento Motor - EDM, com a intenção de comparar tais resultados. Após três meses de intervenção, concluiu-se que a participante apresentou evolução nos aspectos motores analisados do instrumento, tais comoidade motora geral, idade motora do equilíbrio, idade motora do esquema corporal, equilíbrio e o esquema corporal, que até então estavam classificados pelo instrumento como "muito inferior" para sua idade cronológica, igualando-a com o desenvolvimento de uma criança de aproximadamente dois anos. Com os ganhos pós intervenção, o desenvolvimento da criança participante do estudo pode ser comparado com o de outra criança de quatro anos.

No estudo de Oliveira et al. (2015) os autores também investigaram o perfil motor de seis indivíduos com TEA, por meio do mesmo instrumento apresentado no estudo anterior. Foi identificado que duas crianças apresentavam índices maiores na coordenação fina e global e no equilíbrio, em relação ao desenvolvimento motor e que quatro dos indivíduos apresentavam déficits na coordenação motora.

Já em estudo mais recente, Gusman (2017) comparou em dois momentos distintos dez crianças com desenvolvimento típico e dez crianças diagnosticadas com TEA. Identificou-se nos resultados que o segundo grupo de crianças apresentava características peculiares em 
seu desenvolvimento, sendo a hipotonia, déficits na preensão,coordenação motoraealteração no controle motor as mais notadas. Além disso, foi identificado queas crianças com TEA apresentaram resultados abaixo da média manifestando uma menor estabilidade do controle postural, apraxia, surgimento de estereotipias e sensibilidade a ruídos externos, além de oscilações de humor e atenção durante a aplicação do instrumento.

Especificamente quanto ao perfil sensorial, no estudo de Posar\& Visconti (2018), os autores objetivaram fazer uma análise narrativa de estudos já existentes na literatura com a finalidade de resumir, destacar e correlacionar as alterações sensoriais mais comuns em crianças com TEA e suas implicações para este público. Os resultados identificados sinalizam que as alterações encontradas em geral, por mais que sejam características marcantes do TEA, não são específicas do mesmo, já que também são encontradas em crianças com deficiência intelectual. Porém, suas particularidades encontram-se na excessiva ou reduzida responsividade e na constante busca sensorial.

Segundo os autores, as alterações sensoriais também implicam em sintomas de ansiedade e angústia que podem ainda fazer com que as crianças apresentem comportamentos repetitivos e reações muito intensas a determinados estímulos (Posar \& Visconti, 2018).

Diante do exposto, ainda que incipientes, foram encontrados estudos principalmente da última década, que tem se debruçado a investigar essa temática. Compreende-se a relevância de mais estudos que se dediquem a investigar o perfil psicomotor e sensorial de crianças com TEA, de forma a avançar na identificação dos fatores associados ao quadro, contribuindo para uma intervenção ampliada e efetiva, que responda também as demandas psicomotoras e sensoriais apresentada por essa população.

Sendo assim, este estudo tem como objetivo identificar as características psicomotoras e sensoriais de crianças com diagnóstico de TEA atendidas no setor de Terapia Ocupacional na Unidade Saúde Escola da Universidade Federal de São Carlos (USE-UfSCAR).

\section{MÉTOdo}

Trata-se de uma pesquisa exploratória e descritiva,que adota uma abordagem quantitativa, submetida e aprovada pelo Comitê de Ética em Pesquisa (CEP) envolvendo seres humanos, cujas famílias participantes foram devidamente esclarecidas e os responsáveis pelas crianças autorizaram a participação através de um Termo de Consentimento Livre e Esclarecido (TCLE).

Foram participantes da pesquisa nove crianças de quatro a dez anos de idade, com diagnóstico de TEA, vinculados a uma Unidade Saúde Escola (USE), e seus responsáveis. Todo o estudo foi realizado na unidade de atendimento vinculada a uma universidade federal brasileira, caracterizada como um ambulatório de média complexidade abrangendo a população da cidade e região, onde os usuários são atendidos por professores, profissionais da saúde e alunos de graduação e pós-graduação.

A identificação dos participantes ocorreu a partir da lista de crianças inseridas nas ações realizadas no referido serviço pela Terapia Ocupacional no campo da saúde mental infanto-juvenil. Foram aplicados os seguintes critérios de inclusão: crianças entre quatro e dez anos, que mesmo com os comprometimentos advindos do autismo, conseguissem compreender e responder às solicitações da Bateria Psicomotora (вPM).

Foram utilizados três instrumentos para a coleta de dados, escolhidos afim de identificar as características psicomotora se sensórias destas crianças, a saber:

Questionário de dados gerais: foi elaborado pelas pesquisadoras com o objetivo de abordar questões relativas à história de vida e quadro clínico das crianças;

Bateria Psicomotora (BPM): composto por sete fatores psicomotores, sendo tonicidade, equilibração, lateralização, noção do corpo, estruturação espaço-temporal, praxia global e praxia fina, subdivididas em 26 exercícios do dia a dia, pontuados de 1 a 4, sendo que 1 se refere à "realização imperfeita" e 4 à "realização perfeita". Ao final dessas pontuações, os fatores psicomotores são classificados em perfil apráxico, dispráxico, eupráxico e hiperpráxico (Pereira, 2005). Aponta-se que а вРм pode ser aplicada em crianças com idade entre quatro e doze anos. 
Este instrumento foi aplicado pelas pesquisadoras com as instruções do roteiro de aplicação, sendo que o item "tonicidade" foi excluído devido a dificuldades na compreensão de determinados exercíciosa serem realizados pelas crianças com TEA.

Perfil sensorial: É composto por 125 itens separados em 3 seções- processamento sensorial, modulação e comportamento e respostas emocionais (Mattos et al, 2015; Rocha \& Dounis, 2013). É um método de avaliação padronizado para mensurar as habilidades de processamento sensorial de crianças com ou sem deficiência na faixa etária entre três e dez anos (Ermer \& Dunn, 1998).

O Questionário e o Perfil Sensorial foram aplicados em forma de entrevista com os responsáveis pelas crianças. A elaboração e validação dos instrumentos seguiu-se da seguinte forma: O Questionário foi elaborado pela pesquisadora e submetido a duas aplicações piloto. Após a revisão, o instrumento foi adequado para coleta de dados. Os instrumentos perfil sensorial e bateria psicomotora já são padronizados e validados no Brasil, sendo apenas realizada uma aplicação piloto com duas crianças.

Os dados foram coletados ao longo de dois meses em dias e horários agendados com antecedência com os pais/responsáveis pelas crianças. No dia da coleta, a sala era preparada antes da criança chegar, sendo que para alguns testes, foi necessário o uso de objetos e brinquedos providenciados previamente pela pesquisadora. Os registros foram feitos diretamente nos instrumentos. Quanto a coleta de dados com os pais/ responsáveis também ocorreu em salas de consultórios da unidade após a explicação da pesquisadora quanto ao funcionamento dos instrumentos e finalidade.

Os dados obtidos por meio dos instrumentos padronizados foram analisados a partir das instruções préexistentes, e em relação ao Questionário, foi realizada uma análise descritiva dos dados relativos aos participantes.

Assim, no perfil sensorial o resultado foi obtido a partir do escore final de cada seção(processamento sensorial, modulação e comportamento e respostas emocionais), sendo que quanto menor este escore, maior o grau de alteração sensorial da criança, classificando seu desempenho como: típico, diferença provável ou diferença clara (Rocha \& Dounis, 2013).
Na análise da BPM, os exercícios que a compõem são pontuados de 1 a 4 . Ao final de cada fator psicomotor (tonicidade, equilíbrio, lateralização, noção do corpo, estruturação espaço-temporal, praxia global e praxia fina), é somado a pontuação e dividido pelo número de exercícios, criando uma média para este fator, possibilitando classificá-lo em: perfil apráxico (fraco), dispráxico (satisfatório), eupráxico (bom) e hiperpráxico (excelente)(Pereira, 2005).

\section{Resultados}

Os resultados foram divididose serão apresentados de acordo com cada instrumento.

Quanto aos resultados relativos à caracterização dos participantes, todas as crianças eram do sexo masculino. Amédia de idade foide 6,2 anos. Os diagnósticos foram obtidos através de: psiquiatra (4 crianças), equipe multiprofissional (4 crianças) e pediatra ( 1 criança).

Em relação aos aspectos do desenvolvimento psicomotor, de acordo com os responsáveis, todas as crianças correm, pulam, sobem e descem degraus normalmente, sendo que 7 delas apresentam agitação motora.

Os resultados advindos da $\mathrm{BPM}$, dispostos na tabela 1 a seguir,apontaram que 7 crianças $(77,7 \%)$ apresentam desempenho fraco ou satisfatório no item relacionado à praxia global e fina. Resultado semelhante ocorreu em "noção do corpo" e "estruturação espaço-temporal", com 6 crianças $(66,6 \%)$ pontuando 1 ou 2.

Para além dos déficits identificados, o instrumento classifica como "desempenho bom e excelente" as crianças que pontuaram 3 e 4 . Nota-se neste estudo que esse desempenho foi positivo em relação aos itens equilíbrio e lateralidade, sendo 6 crianças $(66,6 \%)$ e 8 crianças $(88,8 \%)$, respectivamente.

Em relação às características sensoriais das crianças participantes, os resultados podem ser visualizados a partir de cada seção do Perfil Sensorial e por meio da grade de fatores, respectivamente nas tabelas 2 e 3 . 
TABELA 1 RESULTADOS DA BATERIA PSICOMOTORA GERAL (BPG), COM PORCENTAGEM DE CRIANÇAS POR PONTUAÇÃO

\begin{tabular}{|c|c|c|c|c|}
\hline & $\begin{array}{c}\mathbf{1} \\
\text { Desempenho } \\
\text { Fraco }\end{array}$ & $\begin{array}{c}\mathbf{2} \\
\text { Desempenho } \\
\text { Satisfatório }\end{array}$ & $\begin{array}{c}\mathbf{3} \\
\text { Desempenho } \\
\text { Bom }\end{array}$ & $\begin{array}{c}\mathbf{4} \\
\text { Desempenho } \\
\text { Excelente }\end{array}$ \\
\hline Equilíbrio & - & $3(33,3 \%)$ & $4(44,4 \%)$ & $2(22,2 \%)$ \\
\hline Lateralização & - & $1(11,1 \%)$ & $2(22,2 \%)$ & $6(66,6 \%)$ \\
\hline Noção do corpo & $3(33,3 \%)$ & $3(33,3 \%)$ & $2(22,2 \%)$ & $1(11,1 \%)$ \\
\hline Estruturação espaço-temporal & $4(44,4 \%)$ & $2(22,2 \%)$ & $1(11,1 \%)$ & $2(22,2 \%)$ \\
\hline Praxia global & $2(22,2 \%)$ & $5(55,5 \%)$ & $1(11,1 \%)$ & $1(11,1 \%)$ \\
\hline Praxia fina & $5(55,5 \%)$ & $2(22,2 \%)$ & $2(22,2 \%)$ & - \\
\hline
\end{tabular}

TABELA 2 RESULTADOS DO PERFIL SENSORIAL POR SEÇÃO

\begin{tabular}{|c|c|c|c|c|}
\hline \multicolumn{2}{|r|}{ Seções do Perfil Sensorial } & $\begin{array}{l}\text { Desempenho } \\
\text { Típico }\end{array}$ & $\begin{array}{l}\text { Diferença } \\
\text { Provável }\end{array}$ & $\begin{array}{l}\text { Diferença } \\
\text { Clara }\end{array}$ \\
\hline \multirow{6}{*}{$\begin{array}{l}\text { Processamento } \\
\text { Sensorial }\end{array}$} & Auditivo & $2(22,2 \%)$ & $4(44,4 \%)$ & $3(33,3 \%)$ \\
\hline & Visual & $9(100 \%)$ & - & - \\
\hline & Vestibular & $4(44,4 \%)$ & $2(22,2 \%)$ & $3(33,3 \%)$ \\
\hline & Tátil & $9(77,7 \%)$ & $1(11,1 \%)$ & $1(11,1 \%)$ \\
\hline & Multissensorial & $3(33,3 \%)$ & $3(33,3 \%)$ & $3(33,3 \%)$ \\
\hline & Oral & $6(66,6 \%)$ & - & $3(33,3 \%)$ \\
\hline \multirow{5}{*}{ Modulação } & $\begin{array}{c}\text { Processamento sensorial relacionado } \\
\text { a tônus }\end{array}$ & $8(88,8 \%)$ & - & $1(11,1 \%)$ \\
\hline & $\begin{array}{l}\text { Modulação relacionada a posição do } \\
\text { corpo no espaço }\end{array}$ & $6(66,6 \%)$ & - & $3(33,3 \%)$ \\
\hline & $\begin{array}{l}\text { Modulação do movimento afetando } \\
\text { nível de atividade }\end{array}$ & $5(55,5 \%)$ & $3(33,3 \%)$ & $1(11,1 \%)$ \\
\hline & $\begin{array}{l}\text { Modulação da entrada sensorial afetando } \\
\text { respostas emocionais }\end{array}$ & $3(33,3 \%)$ & $1(11,1 \%)$ & $5(55,5 \%)$ \\
\hline & $\begin{array}{c}\text { Modulação da entrada visual afetando } \\
\text { respostas emocionais }\end{array}$ & $6(66,6 \%)$ & $2(22,2 \%)$ & $1(11,1 \%)$ \\
\hline \multirow{3}{*}{$\begin{array}{l}\text { Respostas Com- } \\
\text { portamentais e } \\
\text { Emocionais }\end{array}$} & Respostasemocionais/sociais & $4(44,4 \%)$ & $1(11,1 \%)$ & $4(44,4 \%)$ \\
\hline & $\begin{array}{l}\text { Resultados comportamentais do proces- } \\
\text { samento sensorial }\end{array}$ & $2(22,2 \%)$ & $2(22,2 \%)$ & $5(55,5 \%)$ \\
\hline & Itens que indicam limiar de resposta & $7(77,7 \%)$ & $1(11,1 \%)$ & $1(11,1 \%)$ \\
\hline
\end{tabular}


TABELA 3 RESULTADOS DO PERFIL SENSORIAL:GRADE DE FATORES

\begin{tabular}{|c|c|c|c|}
\hline & Desempenho Típico & Diferença Provável & Diferença Clara \\
\hline Procura Sensorial & $2(22,2 \%)$ & $4(44,4 \%)$ & $3(33,3 \%)$ \\
\hline Emocionalmente Reativo & $4(44,4 \%)$ & $2(22,2 \%)$ & $3(33,3 \%)$ \\
\hline Baixa resistência/Tônus & $8(88,8 \%)$ & $1(11,1 \%)$ & - \\
\hline Sensibilidade sensorial oral & $5(55,5 \%)$ & $1(11,1 \%)$ & $3(33,3 \%)$ \\
\hline Inatenção/Distraibilidade & $2(22,2 \%)$ & $1(11,1 \%)$ & $5(55,5 \%)$ \\
\hline Mau registro & $6(66,6 \%)$ & $2(22,2 \%)$ & $1(11,1 \%)$ \\
\hline Sensibilidade Sensorial & $7(77,7 \%)$ & - & $2(22,2 \%)$ \\
\hline Sedentário & $8(88,8 \%)$ & - & $1(11,1 \%)$ \\
\hline Percepção/Motricidade fina & $4(44,4 \%)$ & $4(44,4 \%)$ & $1(11,1 \%)$ \\
\hline
\end{tabular}

Verificou-se que os sistemas sensoriais com maior alteração de processamento sensorial foram o auditivo, o vestibular, o multissensorial e o oral, sendo que 3 crianças (33,3\%) apresentaram "diferença clara". Além disso, se considerarmos a soma entre "diferença clara" e diferença provável", o quantitativo de crianças aumenta.

$5(55,5 \%)$ participantes da pesquisa apontaram "diferença clara" na "modulação da entrada sensorial afetando respostas emocionais" e 3 (33,3\%) na "modulação relacionada a posição do corpo no espaço". Esses dados tornam-se ainda mais significativos se as crianças que apresentaram diferença provável também forem consideradas, totalizando 4 crianças $(44,4 \%)$ com alteração na "modulação do movimento afetando o nível de atividade", 6 (66,6\%) em "modulação da entrada sensorial afetando respostas emocionais" e 3 (33,3\%) em "modulação da entrada visual afetando respostas emocionais".

Além disso, dentre as repercussões das alterações de "Modulação" nas respostas adaptativas produzidas pela criança em seu cotidiano, encontram-se os aspectos sociais e emocionais. Nesse sentido, como mostra a Tabela 2, foram identificadas alterações tanto nas respostas emocionais/sociais (4 crianças com "diferença clara" e 1 com "diferença provável") como nos resultados comportamentais do processamento sensorial (5 crianças com "diferença clara" e 2 com "diferença provável").
A partir do sumário da grade de fatordo instrumento Perfil Sensorial (Tabela 3), destaca-se a procura sensorial, com7 crianças $(77,7 \%$ ) com diferenças provável e clara. No mesmo raciocínio, 5 (55,5\%) apresentaram diferenças no fator "emocionalmente reativo", 4 (44,4\%) em "sensibilidade sensorial oral", 6 (66,6\%) em inatenção/ distraibilidade", e por fim, 5 (55,5\%) em "percepção/ motricidade fina".

\section{DISCUSSÃO}

Assim como identificado nos resultados do presente estudo, a literatura tem sinalizado que as crianças com TEA apresentam características de agitação psicomotora (Posar; Visconti, 2018; Semensato et al., 2010). Posar \& Visconti (2018), ao realizarem uma pesquisa de levantamento sobre alterações psicomotoras e sensoriais no TEA, Os autores sugerem que possivelmente a agitação psicomotora tem relação com as alterações sensoriais, evidenciadas com maior frequência na audição, devido à intensidade com que essas crianças recebem as informações do ambiente externo, causando, neste caso, uma resposta hiper-reativa ou apenas interpretada como agitação.

A pesquisa de Semensato et al (2010), realizada com um grupo de sete pais e mães em um centro de atendimento particular em Porto Alegre, traz a percepção 
da família não só sobre o comportamento de agitação evidente nas crianças com TEA, mas também aborda as possíveis consequências e impactos no cotidiano. Dentre os vários relatos, a agitação é tida como característica própria da criança e como um resultado do meio em que ela vive, ou seja, se a criança convive em um meio turbulento, é natural que a mesma evidencie alteração em seu comportamento. Além disso, os pais relataram que essa agitação durante algumas atividades cotidianas como, por exemplo, o banho e alimentação, é preocupante pois altera a rotina familiar.

Para além da agitação psicomotora e corroborando com os resultados encontrados na BPM, a literatura têm indicado que as crianças com TEA expressam dificuldades no planejamento de movimentos (principalmente na praxia fina), na noção de si mesmas e na estruturação espaço-temporal. Quanto a praxia fina, autores sugerem que bloqueios no movimento de oponência, além de serem evidentes no TEA, podem interferir na preensão e na escrita (Oliveira, 2002; Lima et al, 2014).

Segundo Oliveira (2002), há evidências de que, referente ao item noção do corpo, as crianças com TEA apresentam alterações no esquema corporal, com dificuldade para percorrer as etapas em que este tipicamente se desenvolve, desde a não desvinculação do corpo com o meio, passando pela capacidade de ter uma consciência do seu próprio corpo até, finalmente, apresentar um esquema corporal estruturado, de forma a ter noção de cada parte de si próprio e realizar atividades segmentadas com total controle. Nesse sentido, o autor aponta que tais dificuldades podem prejudicar a locomoção, o controle dos movimentos e o reconhecimento do próprio corpo (Oliveira, 2002).

No que tange a estruturação espaço-temporal, Fernandes (2008) afirma que a criança com TEA não compreende o tempo do corpo, fazendo com que sua movimentação no espaço não tenha limite, nem orientação. $\mathrm{O}$ autor ressalta que a noção do espaço e tempo dependem da construção da noção corporal, anteriormente analisada como frágil, e do desenvolvimento sensorial, que é também afetado nessas crianças.

Conforme apresentado nos resultados, os aspectos sensoriais foram avaliados por meio do Perfil Sensorial, sendo identificado alterações no sistema auditivo, vestibular, o multissensorial e oral. A primeira seção analisada foi o "Processamento Sensorial", que corresponde à avaliação dos diferentes sistemas sensoriais e expressa a relação da organização das respostas corporais, a partir da captação e da interpretação das informações sensoriais advindas do meio externo e do próprio corpo (Shimizu \& Miranda, 2012). Esses resultados reforçam os achados da pesquisa de Posar \& Visconti (2018), já apresentada anteriormente, onde os autores identificaram alterações dentro de todos os aspectos por eles analisados (visual, auditivo, somatosensorial, olfato, propriocepção e oral).

No que se refere a modulação sensorial, Shimizu \& Miranda (2012), consideram-na como a predisposição do indivíduo em aceitar ou ignorar estímulos, além de regular o grau e a natureza das respostas perante um estímulo sensorial para que ele tenha um comportamento adequado no ambiente. São detectadas alterações na modulação sensorial quando há sinais de hiperresponsividade, hiporresponsividade e procura sensorial. Segundo Ben-Sasson et al (2013), alterações na modulação, como as apresentadas nos resultados do presente estudo, tendem a comprometer a participação dos indivíduos em atividades de vida diária e nas interações.

Sobre o item respostas comportamentais e emocionais, Baranek et al. (2006) apresentam alguns dados que se aproximam dos resultados encontrados. Os autores avaliaram respostas comportamentais às experiências sensoriais do dia-a-dia em crianças de 5 meses a 6 anos no contexto social (como o contato físico) e não social (sons altos e texturas). O objetivo dos autores foi caracterizar crianças pequenas com autismo e discriminar padrões de hipo e hiperresponsividade entre aqueles com autismo, deficiências no desenvolvimento ou desenvolvimento típico. A pesquisa foi feita com uma amostra de 258 crianças divididas por diagnósticos: autismo (56), outros atrasos generalizados no desenvolvimento (24), deficiências no desenvolvimento/retardo mental (33), outros atrasos no desenvolvimento (35) e desenvolvimento típico (110), sendo aplicado o instrumento "Questionário de Experiências Sensoriais" com os cuidadores de cada criança. O grupo composto por crianças com autismo apresentou sintomas aumentados em todas as subescalas, com um aumento no padrão de hiporresponsividade a estímulos sensoriais no contexto social em comparação a todos os grupos. Já em âmbito não social, o mesmo grupo apresentou padrão de hiperresponsividade a estímulos sensoriais. 
Os resultados apresentados na Tabela 3 se referem a grade de fator. Na pesquisa de Toreti \&de Medeiros (2015), os autores utilizaram o mesmo instrumento para analisar indivíduos com o diagnóstico de TEA, assim como também, analisaram a grade de fator. Os autores encontraram um maior número de crianças com "diferença clara"nos itens: procura sensorial; emocionalmente reativo; baixa resistência/tônus; Inatenção/distraibilidade e Mau registro. Somando-se essas respostas à "diferença provável", os números aumentam significativamente nos itens: Baixa resistência/tônus;Inatenção/ distraibilidade; Sensibilidade sensorial e Sedentarismo. Dessa forma, comparando-se os resultados encontrados no presente estudo com os da pesquisa de Toreti \& de Medeiros (2015), identifica-se que os mesmos são semelhantes, de forma a reafirmar a presença de tais alterações em indivíduos com TEA.

De modo geral, identificou-se que os resultados encontrados reafirmam o que a literatura tem investigado sobre as características psicomotoras e sensoriais de crianças com tEA. Nessa direção, compreende-se que reconhecer essa realidade e as dificuldades existentes no quadro, pode contribuir para inclusão dessas demandas na prática clínica dos profissionais, favorecendo o desenvolvimento desses indivíduos.

\section{CONSIDERAÇÕES FINAIS}

A partir deste estudo foi possível identificar as características psicomotora e sensoriais de crianças com diagnóstico de TEA atendidas pela Terapia Ocupacional em uma Unidade Saúde Escola, de forma que os participantes apresentam déficits no desenvolvimento psicomotor, principalmente no que se refere a noção espacial/temporal, corporal e coordenação motora global e fina. Já em âmbito sensorial, os déficits mais significativos se encontram no campo do processamento sensorial, mais especificamente nos sistemas auditivo, vestibular, multissensorial e oral,além da inatenção, sensibilidade oral, constante procura sensorial e na modulação sensorial, destacando-se as modulações nas respostas emocionais.

Quanto às limitações, aponta-se para número reduzido de participantes, principalmente devido à dificuldade em incluir crianças elegíveis aos critérios de inclusão e exclusão.
Considera-se essa pesquisa de extrema relevância, pois evidencia as alterações encontradas no quadro de TEA e a necessidade de intervenções mais eficazes e direcionadas para tais demandas. Assim, indica-se a continuidade de estudos dessa natureza, abarcando um maior número de participantes e outros instrumentos de avaliação, uma vez que podem contribuir ainda mais para a compreensão do TEA e suas reais necessidades.

\section{REFERÊNCIAS BIBLIOGRÁFICAS}

American Psychiatric Association. (2013).Diagnostic and statistical manual of mental disorders (DSM-5). $5^{\circ}$ ed. Washington, DC: American Psychiatric Association.

Andrade, M. P. (2012). Autismo e Integração Sensorial: a intervenção psicomotora como um instrumento facilitador no atendimento de crianças e adolescentes com autismo. (Dissertação de Mestrado). Universidade Federal de Viçosa, Minas Gerais, 2012. Recuperado de: https://www.locus.ufv.br/handle/123456789/3479

Baranek, G. T., David, F. J., Poe, M. D., Stone, W. L., \& Watson, L. R. (2006). Sensory Experiences Questionnaire: discriminating sensory features in young children with autism, developmental delays, and typical development. Journal of Child Psychology and Psychiatry, 47(6), 591-601.https://doi.org/10.1111/j.1469-7610.2005.01546.x

Ben-Sasson, A., Soto, T. W., Martínez-Pedraza, F., \& Carter, A. S. (2013). Early sensory over-responsivity in toddlers with autism spectrum disorders as a predictor of family impairment and parenting stress. Journal of Child Psychology and Psychiatry, 54(8), 846-853. https://doi.org/10.1111/jcpp.12035

Brasil (2013).Linha de cuidado para a atenção às pessoas com Transtornos do Espectro do Autismo e suas famílias na rede de atenção psicossocial do Sistema Único de Saúde. Ministério da Saúde.Versão Preliminar, p. 19- 30, Brasília.Recuperado de: https://bvsms.saude.gov.br/ bvs/publicacoes/linha_cuidado_atencao_pessoas_transtorno.pdf

Ermer, J., \& Dunn, W. (1998). The Sensory Profile: A discriminant analysis of children with and without disabilities. The American Journal of Occupational Therapy, 52(4), 283-290.https://doi.org/10.5014/ ajot.52.4.283

Fernandes, F. (2008). Cuerpo en el Autismo. PSIC-revista de Psicologia da Vetor Editora, 9(1), 109-114.Recuperado de: v9n1a13.pdf (bvsalud.org)

Ferreira, A. C. D. (2016). Efeitos de sessões de psicomotricidade relacional sobre o perfil das habilidades motoras e controle postural em indivíduo com transtorno do espectro autista (Dissertação de Mestrado), Universidade Federal do Rio Grande do Norte, Natal, 2016,Brasil. Recuperado de: https://repositorio.unb.br/handle/10482/39441

Gadia, C. A., Tuchman, R., \& Rotta, N. T. (2004). Autismo e doenças invasivas de desenvolvimento. Jornal de pediatria, 80(2), 83-94. https://doi.org/10.1590/S0021-75572004000300011 
Gomes, E., Pedroso, F. S., \& Wagner, M. B. (2008). Hipersensibilidade auditiva no transtorno do espectro autístico. Pró-Fono Revista de Atualização Científica, 20(4), 279-284.http://dx.doi.org/10.1590/ S0104-56872008000400013

Gusman, S. (2017). Aplicação da escala de desenvolvimento motor de Rosa Neto em crianças com Transtorno do Espectro Autista: um estudo exploratório. (Dissertação de Mestrado), Universidade Presbiteriana Mackenzie, São Paulo, Brasil. Recuperado de: http://tede.mackenzie.br/jspui/handle/tede/3350

Klin, A. (2006). Autismo e síndrome de Asperger: uma visão geral. Brazilian Journal of Psychiatry, 28, 3-11.https://doi.org/10.1590/ S1516-44462006000500002

Leal, M., Nagata, M., de Morais Cunha, N., Pavanello, U., \& Ferreira, N. V. R. (2015). Terapia nutricional em crianças com transtorno do espectro autista. Cadernos da Escola de Saúde, 1(13).Recuperado de:https://portaldeperiodicos.unibrasil.com.br/index.php/ cadernossaude/article/view/2425

Lima, A. F. C. D., Gehres, A. D. F., Lorenzini, A. R., \& Brasileiro, L. T. (2017). A Influência de práticas pedagógicas e terapêuticas não verbais no transtorno do espectro autista: as possibilidades para o profissional de educação física. Motricidade, 13, 87-96.http:// dx.doi.org/10.6063/motricidade.12867

Lima, C. R. D. B. V., Afonso, C., Calado, A. C., Torgal, F., Gouveia, R., \& Nascimento, C. (2014). O impacto do programa integrado para o autismo (PIPA). Revista de Psicologia da Criança e do Adolescente. 5(1), 230-244. Recuperado de:rpca_v5_n1_15.pdf (ulusiada.pt)

Mattos, J. C., D’Antino, M. E. F., \& Cysneiros, R. M. (2015). Confiabilidade e evidências de validade do instrumento sensory profile (DUNN, 1999): Revisão de Literatura. Cadernos de Pós-Graduação em Distúrbios do Desenvolvimento, 15(2), 43-51.Recuperado de:https://www.mackenzie.br/fileadmin/ARQUIVOS/Public/6-pos-graduacao/upm-higienopolis/mestrado-doutorado/ disturbios_desenvolvimento/2015/cadernos/2/CONFIABILIDADE_E_EVIDE_NCIAS_DE_VALIDADE_DO_INSTRUMENTO_SENSORY_PROFILE_DUNN_1999__REVISA_O_DE_LITERATURA.pdf

Miccas, C., Vital, A., Famá, M., \& D’Antino, D. A. (2014). Avaliação de funcionalidade em atividades e participação de alunos com transtornos do espectro do autismo. Revista da Associação Brasileira de Psicopedagogia,31(94), 3-10.Recuperado de:v31n94a02. pdf (gn1.link)

Okuda, P. M. (2010). Caracterização do perfil motor de escolares com transtorno autístico. Revista educação especial, 23(38), 443-454. https://doi.org/10.5902/1984686X1462

Oliveira, G. C. (2002) Desenvolvimento da Psicomotricidade. Psicomotricidade: Educação e reeducação num enfoque psicopedagógico. $7^{\circ}$ ed. Petrópolis: Vozes.

Oliveira, R., Silva, A. S., Chaves, G. C., \& Borges-Orientadora, E. A. C. (2015). Perfil Motor de Crianças Autistas Participantes do Atendimento Educacional Especializado (AEE) da Cidade de Porangatu/
GO. In Anais do Congresso de ensino, pesquisa e extensão da UEG II, 1-8.Recuperadode: https://www.anais.ueg.br/index.php/ cepe/article/view/5038/2867

Pereira, K. (2005). Perfil psicomotor: caracterização de escolares da primeira série do ensino fundamental de colégio particular. (Tese de Mestrado). Universidade Federal de São Carlos, São Carlos, São Paulo. Recuperadode: https://repositorio.ufscar.br/handle/ ufscar/5334

Prestes, D. B., Amaro, K. N., Arab, C., \& Neto, F. R. (2009). Avaliação e intervenção motora com uma criança autista. Revista Digital[periódico na Internet], 14(138), 1.Recuperadode: http://www.efdeportes. com/efd138/intervencao-motora-com-uma-crianca-autista.htm

Posar, A., \& Visconti, P. (2018). Sensory abnormalities in children with autism spectrum disorder. Jornal de Pediatria (Versão em português), 94(4), 342-350.https://doi.org/10.1016/j.jpedp.2017.11.009

Rocha, F. D. B., \& Dounis, A. B. (2013). Perfil sensorial de estudantes da primeira série do ensino fundamental: análise e comparação com o desempenho escolar. Cadernos de Terapia Ocupacional da UFSCar (Impr.), 21(2).http://dx.doi.org/10.4322/cto.2013.038

Sandroni, G. A., Ciasca, S. M., \& Rodrigues, S. D. D. (2015). Avaliação da evolução do perfil motor de pré-escolares com necessidades educativas especiais após intervenção psicomotora breve. Revista Psicopedagogia, 32(97), 4-13.Recuperadode:http://pepsic.bvsalud.org/ scielo.php?script=sci_arttext\&pid=S0103-84862015000100002

Semensato, M. R., Schmidt, C., \& Bosa, C. A. (2010). Grupo de familiares de pessoas com autismo: Relatos de experiências parentais. Aletheia, (32), 183-194.Recuperadode: Grupo de familiares de pessoas com autismo: relatos de experiências parentais $\mid$ Semensato | ALETHEIA (ulbra.br)

Shimizu, V. T., \& Miranda, M. C. (2012). Processamento sensorial na criança com TDAH: uma revisão da literatura. Revista Psicopedagogia, 29(89), 256-268. Recuperado de: v29n89a09.pdf (gn1.link)

Soares, A. M., \& Cavalcante Neto, J. L. (2015). Avaliação do comportamento motor em crianças com transtorno do espectro do autismo: uma revisão sistemática. Revista Brasileira de Educação Especial, 21(3), 445-458. https://doi.org/10.1590/S1413-65382115000300010

Toreti, M. S., \& de Medeiros, M. F. D. (2015). Perfil sensorial de crianças com transtorno do espectro do autismo (TEA). Resumo expandido. Anais da XJornada Unisul de Iniciação Científica. Recuperado de: http://rexlab.unisul.br/junic/2015/projeto/projeto_000015.html 